\title{
Composición, diversidad y estructura de la vegetación de bosques ribereños en el centro sur de Chile
}

\section{Composition, diversity and vegetation structure of riparian forests in south-central Chile}

\author{
Ivon Gutierrez ${ }^{\text {a**, Pablo Becerra }}$ a,b \\ *Autor de correspondencia: a Pontificia Universidad Católica de Chile, Facultad de Agronomía e Ingeniería Forestal, \\ Departamento de Ecosistemas y Medio Ambiente, Av. Vicuña Mackenna 4860, Santiago, Chile, \\ tel.: 0051 -958926894, irgutierrez@uc.cl \\ ${ }^{\mathrm{b}}$ Pontificia Universidad Católica de Chile, Center of Applied Ecology and Sustainability (CAPES).
}

\begin{abstract}
SUMMARY
Riparian forests have been highlighted for their unique biological diversity and high importance in ecosystem services. However, they are also ecosystems threatened by forestry, livestock or agriculture. In Chile, studies evaluating the diversity and structure of riparian forest vegetation contrasting slope forests or other habitats are limited. Therefore, the aims of this study were: (1) to compare the composition and diversity of vascular plant species; (2) to compare tree species size structure; and (3) to compare the vertical stratification of plant communities between riparian forests and slope forests. Six southern Chilean watersheds $\left(35-38^{\circ} \mathrm{S}\right)$ were studied, sampling the vegetation in plots of $40 \mathrm{~m}^{2}$. In each watershed, 10 plots were located in riparian forests and 10 in slope forests, on both sides of each river. We found important differences in species composition between the two forest types. In the riparian forest we found a higher diversity of tree and climbing species, higher diversity and cover of native species, higher density of tree species in adult state and higher cover of the strata 1-5 m. In the slope forest we found a larger richness and cover of exotic species, higher regeneration density and higher cover of strata $<1 \mathrm{~m}$ and $>5 \mathrm{~m}$. Results can be related to the higher moisture of riparian forests, showing the particularity of riparian forests regarding species composition, diversity and structure, compared to other habitats.
\end{abstract}

Key words: riparian forests, forests habitats, diversity, structure, regeneration.

\section{RESUMEN}

Los bosques ribereños han sido destacados por presentar una diversidad biológica singular y una alta importancia en servicios ecosistémicos. Sin embargo, son también ecosistemas amenazados por actividades forestales, ganadería o agricultura. En Chile, son limitados los estudios que evalúan la diversidad y estructura de la vegetación de los bosques ribereños en comparación con los de las laderas. Por ello, los objetivos de este estudio fueron: (1) comparar la composición y diversidad de especies de plantas vasculares, (2) comparar la estructura de tamaños de árboles, y (3) comparar la estratificación vertical de comunidades vegetales, entre bosques ribereños y de laderas. Se estudiaron seis cuencas del centro sur de Chile (35-38 $\mathrm{S})$, muestreando la vegetación en parcelas de $40 \mathrm{~m}^{2}$. En cada cuenca se ubicaron 10 parcelas en el bosque ribereño y 10 en el bosque de ladera, a ambos lados de cada río. En el bosque ribereño, se observó mayor diversidad de árboles y plantas trepadoras, mayor diversidad y cobertura de especies nativas, mayor densidad de árboles en estado adulto y mayor cobertura del estrato arbustivo. En el bosque de ladera, se encontró una mayor riqueza y cobertura de especies exóticas, mayor densidad de regeneración y mayor cobertura de los estratos herbáceo y arbóreo. Los resultados pueden relacionarse a la mayor humedad de los bosques ribereños, evidenciando la particularidad de la vegetación de los bosques ribereños en composición, diversidad y estructura, en comparación a los bosques de laderas.

Palabras clave: bosque ribereño, hábitats forestales, diversidad, estructura, regeneración.

\section{INTRODUCCIÓN}

Los bosques ribereños han sido reconocidos a nivel mundial por albergar una alta diversidad de especies de fauna y flora silvestre, especialistas de hábitats húmedos, y ser reguladores de funciones ecosistémicas, especialmente de los ciclos hidrológicos (Naiman y Decamps 1997, Corbacho et al. 2003, Burton et al. 2005, Richardson et al. 2007, Méndez-Toribio et al. 2014). Además, han sido documentados como importantes refugios de la flora regional en épocas secas, actuar como corredores y buffers biológicos, filtro de nutrientes, entre otros (Naiman y Decamps 1997).

Varios trabajos han registrado diferencias importantes en cuanto a la composición, la diversidad y la estructura de las comunidades vegetales cuando se comparan los bosques ribereños con los no ribereños, debido a sus condiciones ambientales particulares de humedad y luz 
(Corbacho et al. 2003) y a la frecuencia de perturbaciones naturales (Berthelot et al. 2014). Por ejemplo, los bosques ribereños normalmente poseen mayor área basal y productividad (Naiman y Decamps 1997, Granados-Sánchez et al. 2006), mayor cobertura de especies arbóreas (Corbacho et al. 2003, Goebel et al. 2003), menor densidad de regeneración de especies arbóreas (Berthelot et al. 2014), mayor diversidad estructural, menor cobertura de plantas herbáceas (Harper y Macdonald 2001), mayor diversidad y equitabilidad de especies (Suzuki et al. 2002, Goebel et al. 2003) y mayor diversidad de fauna silvestre (Naiman y Decamps 1997). Adicionalmente, y probablemente relacionado con estos patrones, los bosques ribereños están sometidos a una alta heterogeneidad de condiciones ambientales, por ejemplo, aquella generada por la amplia variación en la frecuencia de los flujos de agua (Naiman y Decamps 1997), la alta variabilidad topográfica y de los suelos, y los diversos regímenes de perturbaciones originados en las zonas de laderas y en las mismas zonas ribereñas (Richardson et al. 2007).

A pesar de su importancia ambiental, social y económica, los bosques ribereños están entre los ecosistemas más amenazados por las actividades antrópicas (Sambaré et al. 2011), debido a su sensibilidad a los cambios del medio ambiente (Lyon y Gross 2005).

De este modo, actividades asociadas a la agricultura, la ganadería, la urbanización y las plantaciones forestales con especies exóticas, afectan a la biodiversidad y servicios ecosistémicos de estos ecosistemas (Méndez-Toribio et al. 2014). Además, debido a los frecuentes procesos de perturbación, son más susceptibles de sufrir procesos de invasión de especies exóticas (Hood y Naiman 2000, Sunil et al. 2011). Por las razones mencionadas, se han planteado diversas medidas para conservar la biodiversidad de este tipo de ecosistema, tales como, el establecimiento de zonas buffer alrededor de ellos, lo que permite reducir las fuentes de alteración que los puedan afectar (Goebel et al. 2003, Boothroyd et al. 2004, Langer et al. 2008)

En Chile, los estudios sobre las particularidades de los bosques ribereños son limitados (Altamirano y Lara 2010). Se desconoce en qué medida estos difieren en diversidad, composición y estructura de la vegetación en comparación a los aledaños, como los de las laderas. Además, los pocos estudios que se han publicado, comparan ambos tipos de bosque en una misma localidad y no se han hecho estudios que examinen estas diferencias a una mayor escala geográfica, de manera que se puedan obtener resultados generalizables.

Debido principalmente a la mayor disponibilidad hídrica, a la mayor productividad y a la mayor frecuencia de perturbaciones naturales de los bosques ribereños en comparación con los no ribereños (Suzuki et al. 2002, Xiong et al. 2003, Berthelot et al. 2014), se postula que los bosques ribereños debieran tener una composición específica diferente, una mayor diversidad de especies nativas y exóticas, y una mayor densidad y complejidad estructural que los de las laderas. En este contexto, los objetivos de este trabajo son: (1) comparar la composición y diversidad de especies de plantas vasculares, (2) comparar la estructura de tamaños de especies arbóreas, (3) comparar la estratificación vertical de comunidades vegetales, entre bosques ribereños y de laderas.

\section{MÉTODOS}

Área de estudio. El estudio se realizó en la zona centro sur de Chile, entre las regiones del Maule y del Biobío (35-38 $8^{\circ}$ S). Se seleccionaron seis cuencas con vegetación nativa, con similares condiciones climáticas y ecológicas (figura 1). De acuerdo con Gajardo (1994), todos los sitios de estudio corresponden a la región fitoecológica del bosque caducifolio, área de bosque templado dominado por especies caducas tales como Nothofagus obliqua (Mirb.) Oerst., N. glauca (Phil.) Krasser, N. alpina (Poepp. et Endl.) Oerst. y especies siempreverdes tales como N. dombeyi (Mirb.) Oerst., Cryptocarya alba (Molina) Looser, Aextoxicon punctatum Ruiz et Pav., entre otras (cuadro 1).

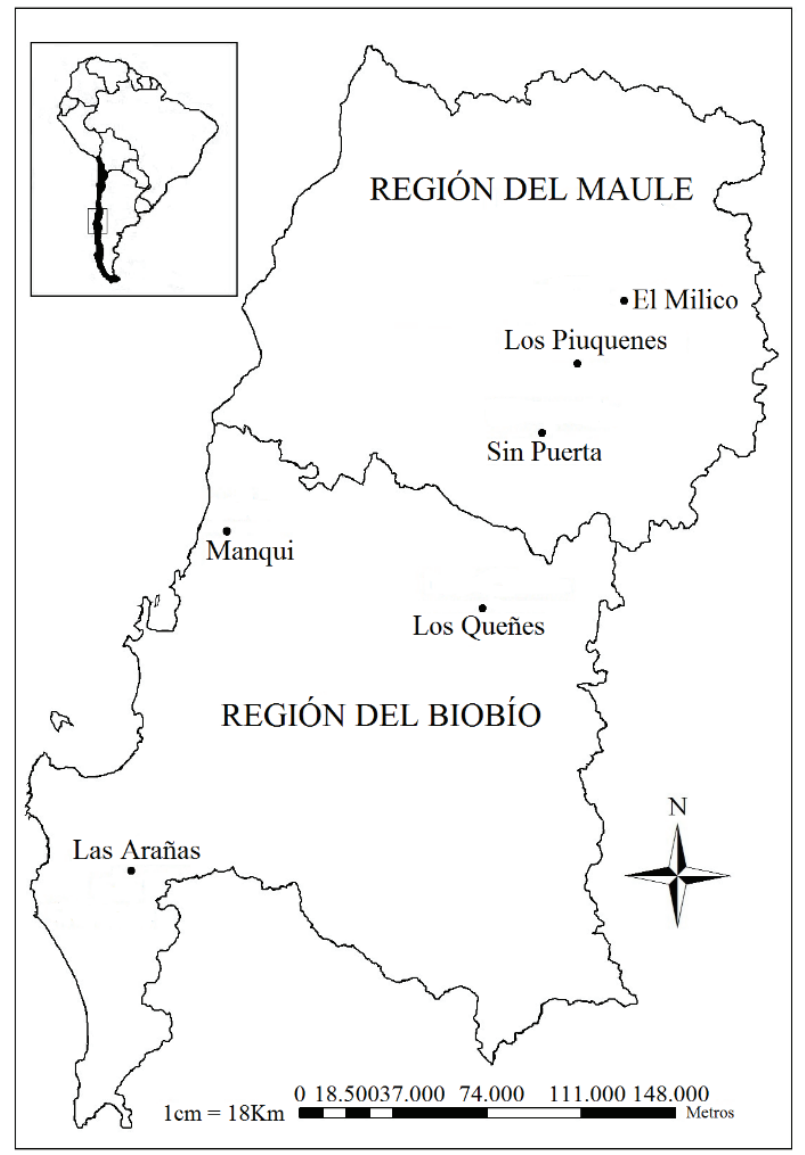

Figura 1. Ubicación de las cuencas de estudio en el centro-sur de Chile, dentro de las regiones del Maule y Biobío.

Location of watersheds included in the study within the Maule and Biobío Regions in south-central Chile. 
Cuadro 1.Localización y características de las cuencas incluídas en el estudio. El clima se caracterizó según Santibáñez y Uribe (1993) y la vegetación según Gajardo (1994).

Location and characteristics of watersheds included in the study. Climate follows Santibáñez and Uribe (1993) and vegetation follows Gajardo (1994).

\begin{tabular}{|c|c|c|c|c|c|c|c|}
\hline \multirow{2}{*}{ Nombre } & \multirow{2}{*}{ Región } & \multirow{2}{*}{ Coordenadas } & \multicolumn{2}{|c|}{ Altitud (m s.n.m.) } & \multirow{2}{*}{ Área (ha) } & \multirow{2}{*}{ Clima } & \multirow{2}{*}{ Vegetación } \\
\hline & & & Máxima & Mínima & & & \\
\hline Los Piuquenes & Maule & $\begin{array}{l}35^{\circ} 48^{\prime} 49^{\prime \prime} \mathrm{S} \\
71^{\circ} 11^{\prime} 06^{\prime \prime} \mathrm{O}\end{array}$ & 2.087 & 660 & $2.087,9$ & $\begin{array}{l}\text { Templado mesotermal } \\
\text { inferior estenotérmico } \\
\text { mediterráneo semiárido }\end{array}$ & $\begin{array}{l}\text { Bosque } \\
\text { caducifolio de la } \\
\text { Montaña }\end{array}$ \\
\hline Las Arañas & Biobío & $\begin{array}{l}37^{\circ} 33^{\prime} 33^{\prime \prime} \mathrm{S} \\
73^{\circ} 13^{\prime} 20^{\prime \prime} \mathrm{O}\end{array}$ & 982 & 560 & 142,4 & $\begin{array}{l}\text { Templado infratermal } \\
\text { estenotérmico mediterráneo } \\
\text { perhúmedo }\end{array}$ & $\begin{array}{l}\text { Bosque } \\
\text { caducifolio de } \\
\text { Concepción }\end{array}$ \\
\hline Los Queñes & Biobío & $\begin{array}{l}36^{\circ} 39^{\prime} 33^{\prime \prime} \mathrm{S} \\
71^{\circ} 34^{\prime} 35^{\prime \prime} \mathrm{O}\end{array}$ & 1.561 & 565 & $1.021,6$ & $\begin{array}{l}\text { Templado mesotermal infe- } \\
\text { rior estenotérmico mediter- } \\
\text { ráneo semiárido }\end{array}$ & $\begin{array}{l}\text { Bosque } \\
\text { caducifolio de la } \\
\text { Montaña }\end{array}$ \\
\hline Manqui & Biobío & $\begin{array}{l}36^{\circ} 22^{\prime} 15^{\prime \prime} \mathrm{S} \\
72^{\circ} 44^{\prime} 27^{\prime \prime} \mathrm{O}\end{array}$ & 571 & 78 & 492,3 & $\begin{array}{l}\text { Templado mesotermal } \\
\text { estenotérmico mediterráneo } \\
\text { subhúmedo }\end{array}$ & $\begin{array}{l}\text { Bosque } \\
\text { caducifolio } \\
\text { Maulino }\end{array}$ \\
\hline Sin Puerta & Maule & $\begin{array}{l}36^{\circ} 02^{\prime} 46^{\prime \prime} \mathrm{S} \\
71^{\circ} 19^{\prime} 21^{\prime \prime} \mathrm{O}\end{array}$ & 1.153 & 520 & 235,0 & $\begin{array}{l}\text { Templado mesotermal } \\
\text { estenotérmico mediterráneo } \\
\text { subhúmedo }\end{array}$ & $\begin{array}{l}\text { Bosque } \\
\text { caducifolio } \\
\text { Maulino }\end{array}$ \\
\hline El Milico & Maule & $\begin{array}{l}35^{\circ} 35^{\prime} 18^{\prime \prime} \mathrm{S} \\
71^{\circ} 01^{\prime} 02^{\prime \prime} \mathrm{O}\end{array}$ & 2.282 & 1.496 & 129,6 & $\begin{array}{l}\text { Templado mesotermal } \\
\text { inferior estenotérmico } \\
\text { mediterráneo semiárido }\end{array}$ & $\begin{array}{l}\text { Bosque } \\
\text { caducifolio de la } \\
\text { Montaña }\end{array}$ \\
\hline
\end{tabular}

Diseño de muestreo y variables analizadas. El muestreo se realizó entre agosto del 2013 y enero del 2015. Todas las cuencas que se evaluaron tenían un desnivel, de manera que se separó entre bosque ribereño y de ladera. Para caracterizar la vegetación del bosque ribereño, en cada cuenca se establecieron cinco parcelas de $10 \times 4 \mathrm{~m}$ a cada lado del río (10 en total) y a una distancia no mayor a $5 \mathrm{~m}$ del borde de cada río (ancho promedio de cada río $5 \mathrm{~m}$ ). $\mathrm{La}$ primera parcela fue ubicada en forma aleatoria, las demás parcelas fueron ubicadas en forma sistemática cada $40 \mathrm{~m}$ a lo largo del río. Para caracterizar la vegetación del bosque de ladera, se ascendió $50 \mathrm{~m}$ en distancia lineal desde los ríos y se estudiaron cinco parcelas de $10 \times 4 \mathrm{~m}$ a cada lado del río, también distanciadas por $40 \mathrm{~m}$, siempre a unos 50 $\mathrm{m}$ del río.

Diversidad de especies. En cada parcela se registró la composición de especies de plantas vasculares y el porcentaje de cobertura de cada especie. El porcentaje de cobertura se evaluó mediante una estimación visual (e.g. Pauchard y Alaback 2004). Para ello, primero se registraron las especies que había en la parcela, y luego ubicándose en su parte central, se estimó el porcentaje de cobertura de cada una. En cada cuenca se hizo un análisis de similitud de especies entre ambos tipos de bosque (ribereño y de ladera) empleando el índice de Jaccard (e.g. Goebel et al. 2003). Empleando los datos de cobertura visual se calculó el índice de Shannon-Wiener $(\mathrm{H})$ como una medida de la diversidad (e.g. Lyon y Gross 2005, Gutierrez y Becerra 2017). El cálculo de diversidad y riqueza se desarrolló por tipo de crecimiento (árbol, arbusto, hierba anual o perenne y plantas trepadoras) y por parcela. Respecto a las plantas trepadoras, se incluyeron a especies leñosas (lianas) y a herbáceas; las especies epífitas y las hemiparásitas, se consideraron como plantas trepadoras. Considerando el origen biogeográfico, nativo o exótico, se analizó la riqueza y la cobertura total por parcela. Para el análisis de la diversidad o de la cobertura y de la riqueza de especies, además de incluir a las especies que pudieron ser identificadas, se incluyeron aquellas no identificadas para las que se pudo determinar la forma de vida o el origen biogeográfico. Para la nomenclatura de las especies se siguió a Marticorena y Quezada (1985) y a Tropicos (2018) para las actualizaciones. La clasificación de las especies fue realizada por un taxónomo especialista. Como árbol se consideró a las especies con potencial de crecimiento $>5 \mathrm{~m}$. Los helechos fueron clasificados como plantas herbáceas.

Estructura de tamaños. Para evaluar la estructura de tamaños de los árboles, en cada parcela se registró el diámetro a la altura de pecho (dap, $\sim 1,30 \mathrm{~m}$ de altura) de todos los 
individuos mayores que $2 \mathrm{~m}$ de altura y dap $\geq 5 \mathrm{~cm}$, a los que se les consideró como adultos. Los individuos con dap $<5 \mathrm{~cm}$ fueron clasificados como regeneración. La densidad y riqueza de especies arbóreas por clase de tamaño se comparó entre el bosque ribereño y de ladera.

Estratificación y hojarasca. Se evaluó a través de la medición de cobertura de la vegetación a diferentes alturas. En cada parcela se registró la cobertura de la vegetación según su altura: $<1 \mathrm{~m}$ (herbáceo), 1-5 m (arbustivo) y $>5 \mathrm{~m}$ (arbóreo), con el método de intercepto de puntos, empleando una grilla de 28 puntos ubicados cada $1 \mathrm{~m}$ de distancia a lo largo del contorno de cada parcela. Así, se obtuvo un valor de cobertura en cada parcela por cada clase de altura, donde el intercepto en los 28 puntos representa $100 \%$ de cobertura, mientras que el intercepto en 0 puntos representa $0 \%$ de cobertura. En cada estrato, solo se registró la presencia o ausencia de vegetación, sin hacer distinción entre los tipos de crecimiento. La hojarasca también se evaluó alrededor de la grilla de 28 puntos y de este modo obtener su cobertura. Para medir la profundidad de hojarasca, se utilizó una regla metálica que fue insertada dentro de la hojarasca hasta el nivel del suelo.

Análisis de datos. Las comparaciones de las variables analizadas, entre el bosque ribereño y de ladera, se llevaron a cabo utilizando modelos lineales generalizados (GLM). Los análisis fueron hechos en un diseño anidado, donde el tipo de bosque (ribereño y de ladera) fue considerado como el factor principal, y las cuencas estudiadas fueron anidadas en cada tipo de bosque. Para los datos de diversidad de Shannon se asumió una distribución de tipo gaussiana y función de "identidad", mientras que para los datos de riqueza de especies, densidad, profundidad de hojarasca y coberturas se asumió una distribución de Poisson y función "raíz cuadrada". Todos los análisis se realizaron en el programa estadístico R versión 3.1.2. Los datos de similitud de especies fueron analizados con la prueba $\mathrm{t}$ - Student para una muestra, comparándolos estadísticamente con un valor de completa similitud (1), que corresponde al máximo valor de similitud del índice de Jaccard. Estos análisis se realizaron en el paquete estadístico PAST 3.06 (Paleontological Statistic).

\section{RESULTADOS}

Composición y diversidad de especies. En el bosque ribereño se registraron 160 especies, pertenecientes a 118 géneros y 77 familias. El 35,63 \% (57) fueron hierbas perennes o anuales, $28,75 \%$ (46) arbustos, $21,88 \%$ (35) árboles y $13,75 \%$ (22) plantas trepadoras. En el bosque de ladera se registraron 131 especies, pertenecientes a 102 géneros y 62 familias. El 34,35\% (45) fueron hierbas perennes o anuales, 27,48 \% (36) arbustos, 23,66 \% (31) árboles y $14,50 \%$ (19) plantas trepadoras (anexo). Por otro lado, en el bosque ribereño, de las 155 especies con origen cono- cido, el 5,16\% (8 especies) fueron exóticas asilvestradas, mientras que en el bosque de ladera, de las 129 especies con origen conocido, el 10,85 \% (14 especies) fueron exóticas asilvestradas, principalmente hierbas (78,57 \%).

En el bosque ribereño, las especies presentes en al menos cuatro de las cuencas fueron Aextoxicon punctatum, Aristotelia chilensis (Molina) Stuntz, Citronella mucronata (Ruiz et Pav.) D. Don, Cryptocarya alba, Drimys winteri J.R.Forst. et G.Forst, Gevuina avellana Molina, Laurelia sempervirens (Ruiz et Pav.) Tul., Lomatia dentata (Ruiz et Pav.) R.Br., Luma apiculata (DC.) Burret, N. obliqua, Persea lingue (Ruiz et Pav.) Nees, Chusquea quila Kunth, Rhaphithamnus spinosus (Juss.) Moldenke, Rubus ulmifolius Schott, Viola portalesia Gay, Adiantum chilense Kaulf., Blechnum hastatum Kaulf., Equisetum bogotense Kunth, Bomarea salsilla (L.) Herb., Boquila trifoliolata (DC.) Decne., Hydrangea serratifolia (Hook. et Arn.) F. Phil., Lapageria rosea Ruiz et Pav., Lardizabala biternata Ruiz et Pav. y Luzuriaga radicans Ruiz et Pav. Por otro lado, en el bosque de ladera, las especies más frecuentes fueron A. punctatum, A. chilensis, C. alba, G. avella$n a, L$. sempervirens, $L$. dentata, L. apiculata, $N$. obliqua, P. lingue, C. quila, B. hastatum, Eryngium paniculatum Cav. et Dombey ex F. Delaroche, Cissus striata Ruiz et Pav. y L. biternata.

El índice de similitud promedio entre los tipos de bosque (ribereño y de ladera) fue 0,42 . Los valores de similitud registrados son significativamente menores al nivel de similitud máximo que es $1(\mathrm{t}=23,81, P<0,0001)$. Así mismo, los índices de similitud para los tipos de crecimiento arbustos, hierbas y plantas trepadoras son inferiores al $65 \%$. Específicamente para el caso de hierbas y plantas trepadoras, los índices de similitud fueron los más bajos (cuadro 2), indicando fuertes diferencias en composición de especies.

Como era de esperar, los resultados de similitud indican que la composición de especies del bosque ribereño es diferente del bosque de ladera. Por ejemplo, especies como Amomyrtus luma (Molina) D. Legrand et Kausel, Blepharocalyx cruckshanksii (Hook. et Arn.) Nied., Kageneckia oblonga Ruiz et Pav., Legrandia concinna (Phil.) Kausel, Luma chequen (Molina) A. Gray, Myrceugenia obtusa (DC.) O. Berg., Buddleja globosa Hope, Calceolaria Cf. talcana Grau et C. Ehrhart, Colliguaja odorifera Molina, Coriaria ruscifolia L., Selkirkia pauciflora (Ruiz et Pav.) Holstein et Weigend, Jovellana violacea (Cav.) G. Don., Jovellana punctata Ruiz et Pav., Adiantum sulphureum Kaulf., Cheilanthes hypoleuca (Kunze) Mett., Francoa appendiculata Cav., Libertia tricocca Phil., Loasa tricolor Ker Gawl., Solaria miersioides Phil., Asteranthera ovata (Cav.) Hanst., Campsidium valdivianum (Phil.) Skottsb., Herreria stellata Ruiz et Pav., Lepidoceras chilense (Molina) Kuijt, crecían sólo en el bosque ribereño. En contraste, especies como Eucryphia cordifolia Cav., Nothofagus pumilio (Poepp. et Endl.) Krasser, Adesmia elegans Clos, Baccharis racemosa (Ruiz et Pav.) DC., Misodendrum punctulatum Banks ex DC., Ribes valdivianum Phil., Azorella spinosa 
(Ruiz et Pav.) Pers., Cirsium vulgare (Savi) Ten., Geranium core-core Steud., Hypericum caespitosum Cham. et Schltdl., Quinchamalium chilense Molina, Sanguisorba minor Scop., Uncinia phleoides (Cav.) Pers., Dioscorea humifusa Poepp., Mutisia brachyantha Phil., Mutisia decurrens Cav., lo hacían sólo en el bosque de ladera.

Se encontró una mayor diversidad de árboles $(P=0,003)$ y plantas trepadoras $(P=0,008)$ en el bosque ribereño, pero no hubo diferencias significativas de la diversidad de arbus- tos $(P=0,068)$ y hierbas anuales o perennes $(P=0,173)$. Así mismo, se encontró una mayor riqueza de hierbas anuales y perennes $(P=0,0009)$ y plantas trepadoras $(P<0,0001)$ en el bosque ribereño, mientras que para el caso de la riqueza de árboles $(P=0,761)$ y arbustos $(P=0,085)$ no se encontraron diferencias (figura 2).

En el bosque ribereño en comparación al bosque de ladera, se detectó una mayor cobertura de especies nativas $(P<0,0001)$ y una menor de exóticas asilvestradas

Cuadro 2. Índices de similitud (Jaccard) de la vegetación entre el bosque ribereño y de ladera en cada cuenca de evaluación, considerando el total de especies y tipo de crecimiento.

Similarity indices (Jaccard) of vegetation between riparian and slope forest in each watershed, separately for the total number of species and per growth type.

\begin{tabular}{lccccc}
\hline Cuencas & Total & Árboles & Arbustos & Hierbas & Plantas trepadoras \\
\hline Los Piuquenes & 0,42 & 0,65 & 0,5 & 0,28 & 0,27 \\
Las Arañas & 0,34 & 0,51 & 0,34 & 0,21 & 0,21 \\
Los Queñes & 0,37 & 0,56 & 0,49 & 0,14 & 0,39 \\
Manqui & 0,47 & 0,56 & 0,39 & 0,29 & 0,61 \\
Sin Puerta & 0,50 & 0,57 & 0,45 & 0,34 & 0,60 \\
El Milico & 0,42 & 0,63 & 0,56 & 0,18 & 0,00 \\
\hline
\end{tabular}
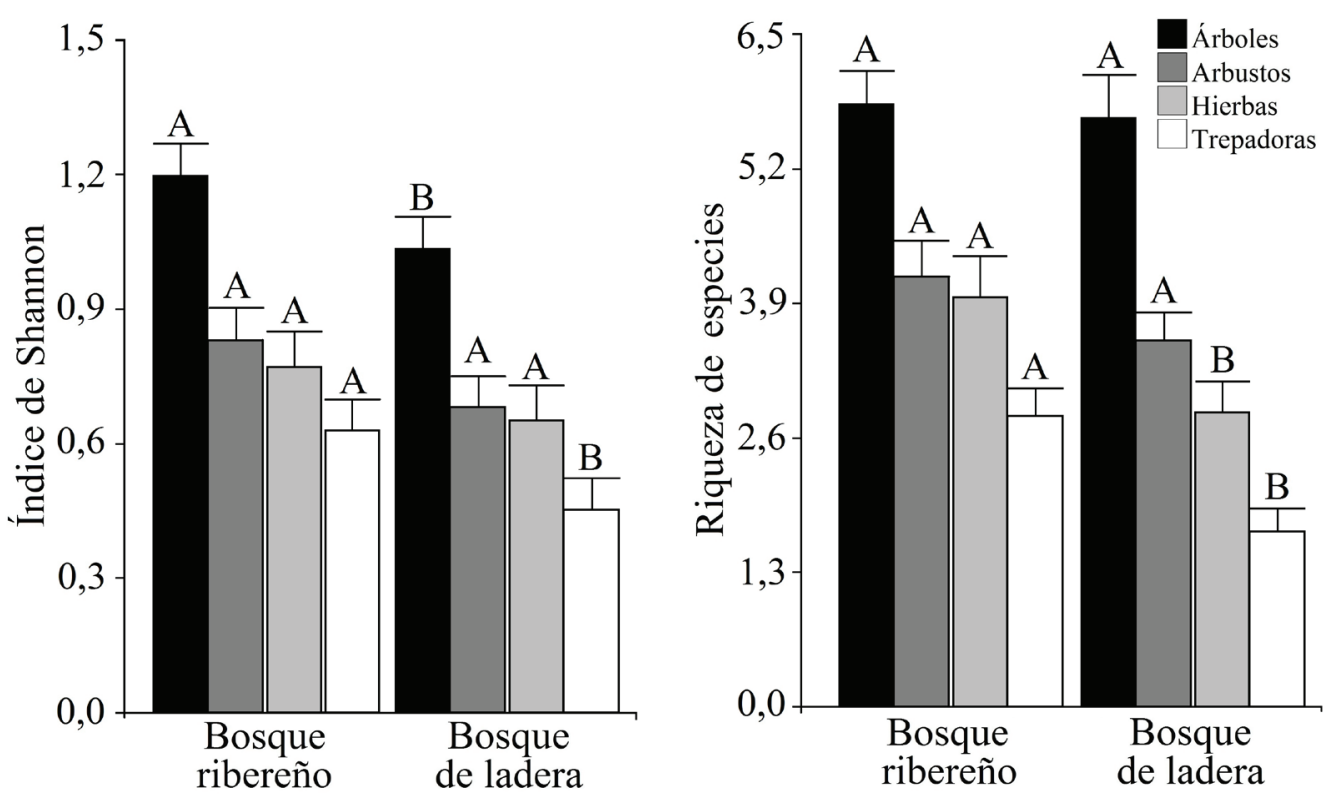

Figura 2. Diversidad de especies (índice de Shannon/parcela) y riqueza de especies/parcela por tipo de crecimiento (árboles, arbustos, hierbas anuales o perennes y plantas trepadoras) en el bosque ribereño y de ladera (media $\pm 1 \mathrm{EE})$. N por cada barra $=60$. Letras diferentes indican diferencias significativas $(P>0,05)$ entre el bosque ribereño y de ladera para un solo tipo de crecimiento.

Species diversity (Shannon index)/plot and species richness/plot per growth type (trees, shrubs, herbs and climbers) in riparian and slope forests (mean $\pm 1 \mathrm{SE}$ ). $\mathrm{N}$ for each bar $=60$. Different letters indicate statistical differences $(P<0.05)$ between riparian and slope forests for a single growth type. 
$(P<0,0001)$. Igualmente, se encontró una mayor riqueza de especies nativas $(P<0,0001)$, pero menor riqueza de exóticas asilvestradas en el bosque ribereño en comparación con el de ladera (figura 3).

Estructura de tamaños de especies arbóreas. Se encontró una mayor densidad de individuos adultos (dap $\geq 5 \mathrm{~cm}$ ) $(P<0,0314)$ y menor densidad de regeneración $($ dap $<5 \mathrm{~cm})$ $(P<0,0001)$ en el bosque ribereño en comparación al de ladera. Igualmente, se encontró una mayor riqueza de árboles en estado adulto $(P=0,0005)$ en el bosque ribereño, pero no se registró ninguna diferencia a nivel de la regeneración $(P=0,187)$ (figura 4$)$.

A nivel de individuos adultos, en el bosque ribereño algunas de las especies con mayor densidad ( $\geq 7$ individuos/parcela) fueron Aextoxicon punctatum, Cryptocarya alba, Caldcluvia paniculata (Cav.) D. Don, Luma apiculata y Nothofagus dombeyi. En cambio, en el bosque de ladera las especies con mayor densidad ( $\geq 7$ individuos/ parcela) fueron Cryptocarya alba, Nothofagus glauca y Persea lingue.

En estado de regeneración, en el bosque ribereño algunas de las especies con mayores niveles de regeneración ( $\geq 50$ individuos/parcela) fueron Aextoxicon punctatum, Cryptocarya alba, Caldcluvia paniculata, Luma apiculata, Lomatia dentata, Maytenus boaria Molina, Nothofagus dombeyi y Persea lingue. En el bosque de ladera, las especies con mayores niveles de regeneración ( $\geq 50$ individuos/ parcela) fueron Aextoxicon punctatum, Cryptocarya alba, Gevuina avellana, Luma apiculata, Lomatia hirsuta (Lam.) Diels, Nothofagus glauca, Nothofagus dombeyi, Nothofagus obliqua, Persea lingue y Peumus boldus Molina.
Estratificación. Se encontró una mayor cobertura del estrato herbáceo (altura $<1 \mathrm{~m})(P=0,012)$ y arbóreo (altura $>5 \mathrm{~m})(P<0,0001)$ en el bosque de ladera, mientras que para el caso del estrato arbustivo (altura 1-5 m), la cobertura fue mayor en el bosque ribereño $(P<0,0001)$ (figura 5). En ambos bosques existe una dominancia del estrato herbáceo; sin embargo, en el bosque ribereño la cobertura del estrato arbustivo alcanza también una alta cobertura en comparación al estrato arbóreo. En cambio, en el bosque de ladera, los dos estratos superiores poseen una menor cobertura que el estrato más bajo (figura 5).

Respecto a la hojarasca, se encontró una mayor cobertura $(P<0,0001)$ y mayor profundidad $(P<0,0001)$ en el bosque de ladera. En el bosque ribereño se encontró $2,76 \pm$ $0,18 \mathrm{~cm}$ de profundidad y $85,42 \pm 2,31 \%$ de cobertura de hojarasca, mientras que en el bosque de ladera se encontró $5,16 \pm 1,62 \mathrm{~cm}$ de profundidad y $96,67 \pm 0,63 \%$ de cobertura de hojarasca.

\section{DISCUSIÓN}

En concordancia con la hipótesis planteada, la composición de especies de plantas vasculares del bosque ribereño y de ladera fue diferente, habiendo especies que se restringen al bosque ribereño y otras que se restringen al bosque de ladera. Estos resultados concuerdan con el estudio de Ramírez et al. (1983), quienes en función de las condiciones edáficas y principalmente de la humedad caracterizan diferentes unidades fitosociológicas. Si bien en todos los tipos de crecimiento evaluados, no alcanzan más de un $65 \%$ de similitud; en particular, es entre las hierbas y plantas trepadoras, donde los tipos de bosque son más diferentes.
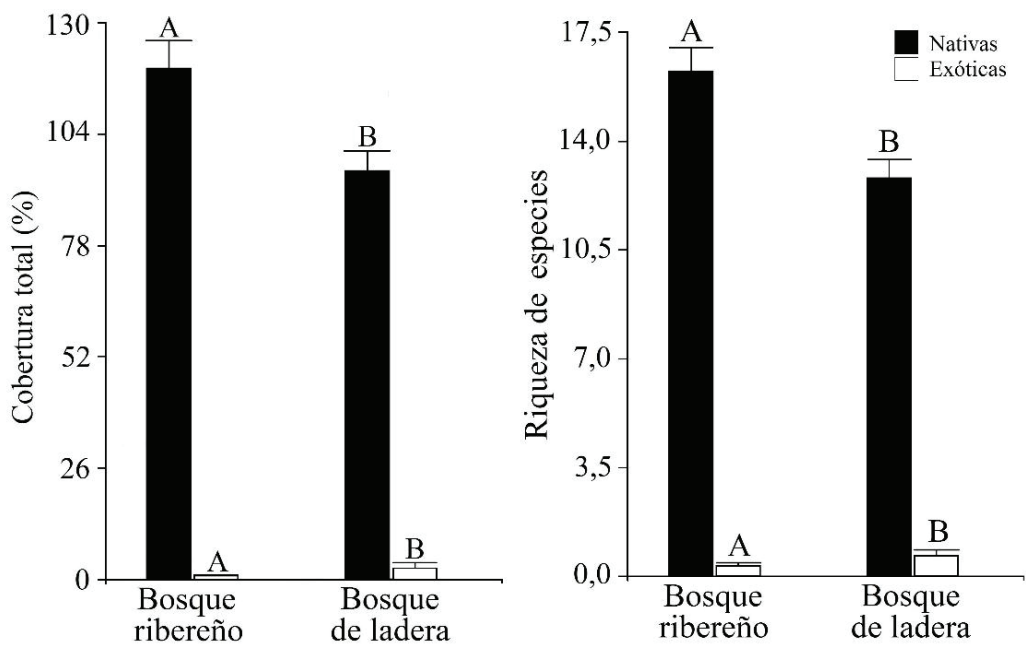

Figura 3. Cobertura total y riqueza de especies/parcela según origen biogeográfico (nativa, exótica) en el bosque ribereño y de ladera (media $\pm 1 \mathrm{EE}) . \mathrm{N}$ por cada barra $=60$. Letras diferentes indican diferencias significativas $(P>0,05)$ entre el bosque ribereño y de ladera por cada origen biogeográfico.

Total cover and species richness/plot according to biogeographic origin (native and exotic) in riparian and slope forests (media $\pm 1 \mathrm{SE}$ ). $\mathrm{N}$ for each bar $=60$. Different letters indicate statistical differences $(P<0.05)$ between riparian and slope forest for a single biogeographic origin. 

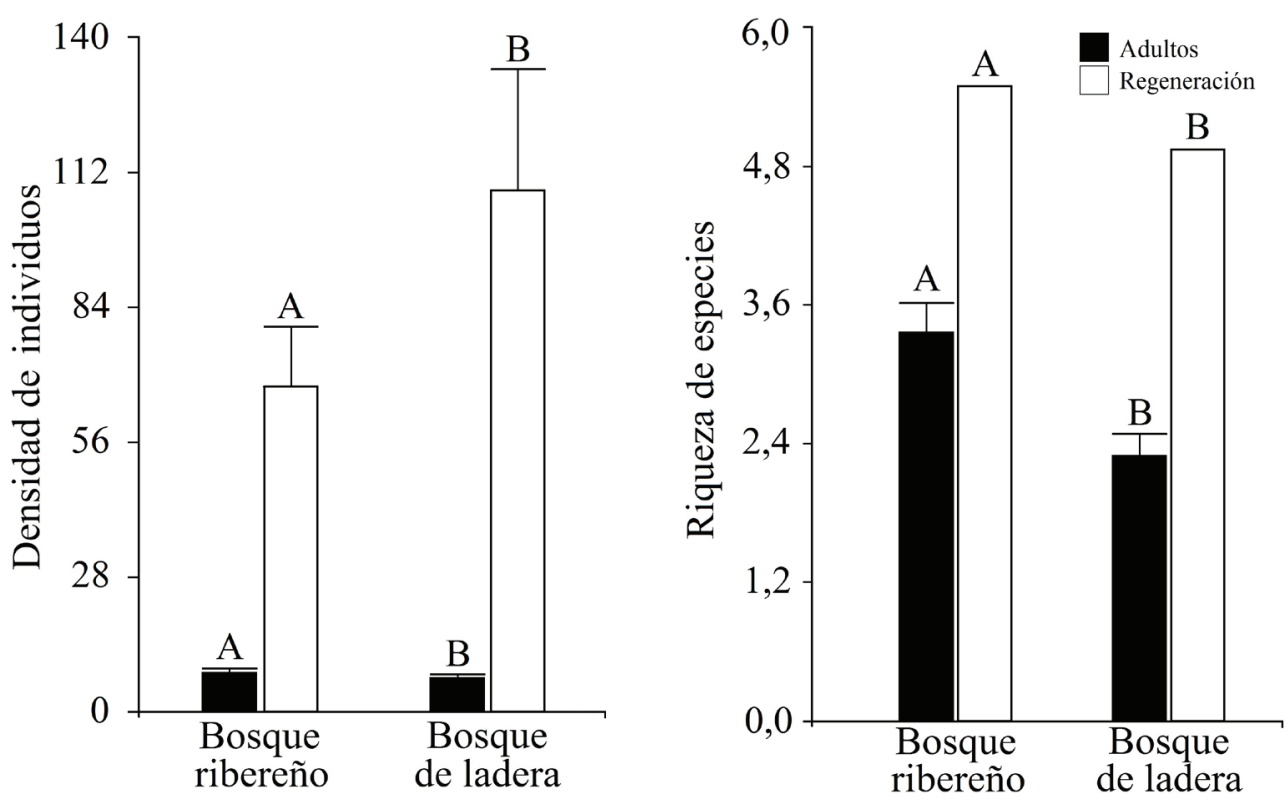

Figura 4. Densidad (individuos/parcela) y riqueza de especies/parcela de árboles en etapa adulta y regeneración en el bosque ribereño y de ladera (media $\pm 1 \mathrm{EE})$. $\mathrm{N}$ por cada barra $=60$. Letras diferentes indican diferencias significativas $(P>0,05)$ entre el bosque ribereño y de ladera por cada clase de tamaño.

Density (individuals/plot) and species richness/plot of tree species for adult and regeneration stages in riparian and slope forests (mean $\pm 1 \mathrm{SE}$ ). $\mathrm{N}$ for each $\mathrm{bar}=60$. Different letters indicate statistical differences $(P<0.05)$ between riparian and slope forests.

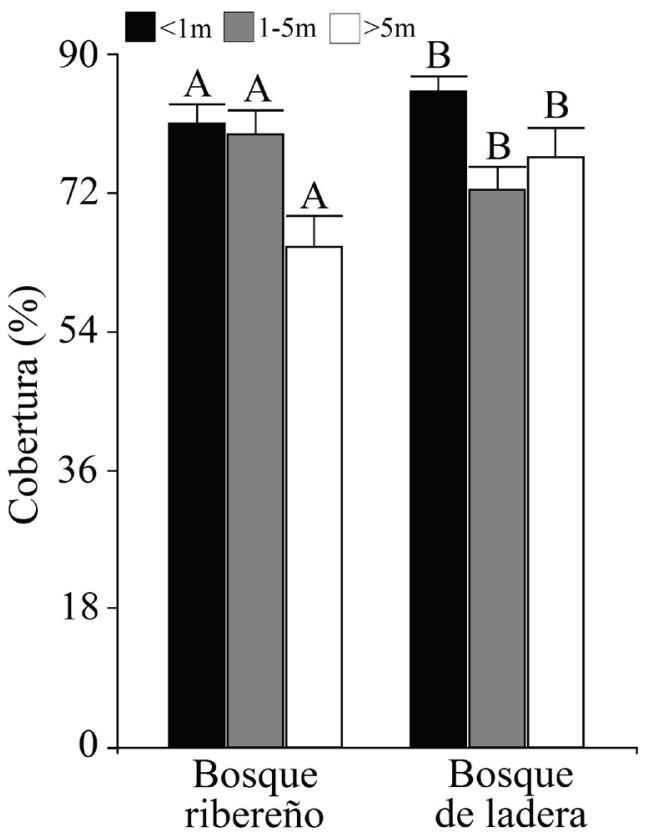

Figura 5. Cobertura de vegetación de tres estratos verticales de altura: $<1 \mathrm{~m}$ (herbáceo), entre $1-5 \mathrm{~m}$ (arbustivo) y $>5 \mathrm{~m}$ (arbóreo) en el bosque ribereño y de ladera (media $\pm 1 \mathrm{EE}$ ). $\mathrm{N}$ por cada barra $=60$. Letras diferentes indican diferencias significativas $(P<0,05)$ entre el bosque ribereño y de ladera para un solo estrato.

Cover of three vertical strata $<1$ (herbaceous), $1-5$ (shrubby) and $>5 \mathrm{~m}$ (arboreal) in riparian and slope forests (mean $\pm 1 \mathrm{SE})$. $\mathrm{N}$ for each bar $=60$. Different letters indicate statistical differences $(P<0.05)$ between riparian and slope forests for a single strata.
En el bosque ribereño se encontró una mayor diversidad de especies, al menos en árboles y plantas trepadoras, una mayor cobertura y riqueza de especies nativas, una mayor densidad de árboles adultos (dap $>5 \mathrm{~cm}$ ) y una menor cobertura del estrato herbáceo (altura $<1 \mathrm{~m}$ ). En cambio, en el bosque de ladera se encontró una mayor riqueza y cobertura de especies exóticas asilvestradas, una mayor densidad de regeneración de árboles y una mayor cobertura del estrato herbáceo (altura $<1 \mathrm{~m}$ ) y arbóreo (altura $>5 \mathrm{~m}$ ).

Aunque no se evaluaron las condiciones abióticas de ambos tipos de bosque, es posible que al menos algunas de estas diferencias florísticas y de vegetación se deban a la mayor disponibilidad de humedad en el hábitat ribereño (e.g. Suzuki et al. 2002, Xiong et al. 2003, Sambaré et al. 2011). Una mayor disponibilidad de agua en el suelo podría reducir la competencia; y con ello, favorecer la coexistencia de un mayor número de especies, una mayor densidad de plantas y una mayor cantidad de biomasa o cobertura, tal como se observó. Esto sería especialmente importante durante los períodos de verano, en los que en la región de estudio, las precipitaciones disminuyen considerablemente. Por ello, una mayor humedad en los bosques ribereños podría explicar la mayor densidad de árboles adultos, la mayor cobertura y la mayor riqueza de especies nativas en comparación con los de la ladera (GranadosSánchez et al. 2006).

Por otra parte, la mayor riqueza de hierbas en el bosque de ladera es consecuencia de una mayor invasión de especies exóticas asilvestradas, que son predominantemen- 
te herbáceas. Así mismo, la mayor cobertura del estrato herbácea en el bosque de ladera, está determinada fuertemente por la mayor cobertura de las especies exóticas que crecen en ellos. Si bien en el bosque de ladera se observó una menor cobertura del estrato arbustivo, hubo mayor cobertura del estrato arbóreo, haciendo que en general la cobertura total de ambos hábitats sea relativamente similar. En consecuencia, la mayor riqueza de especies exóticas, principalmente herbáceas, observada en el bosque de ladera no se debería a diferencias de cobertura leñosa tal como se ha documentado en otros trabajos (Rojas et al. 2011). Es conocido que la invasión por hierbas exóticas asilvestradas es favorecida por la dispersión que genera el ganado (Pauchard y Alaback 2004). Entonces, es posible que el mejor acceso que tienen los bosques de ladera para el ganado, en comparación con los fondos de las quebradas donde crece el bosque ribereño, pueda estar gatillando una mayor presencia de hierbas exóticas. Además, las laderas se encontrarían en general más cercanas a los asentamientos humanos, los que juegan un rol importante en la invasión de especies exóticas (Burton et al. 2005).

La menor densidad, pero no menor riqueza, de regeneración de árboles en el bosque ribereño puede estar relacionada con la mayor cobertura del estrato arbustivo en este tipo de bosque. Este estrato probablemente reduce fuertemente la disponibilidad de luz, restringiendo o reduciendo la densidad de regeneración de especies menos tolerantes a la sombra. Además, el mayor encajonamiento de los bosques ribereños determina que en general estos hábitats reciban menos radiación directa que los bosques de ladera (Granados-Sánchez et al. 2016). En cambio, en el bosque de ladera la menor cobertura del estrato arbustivo, pero al mismo tiempo manteniendo una cobertura superior arbórea que protege de la radiación directa, puede permitir una alta regeneración tanto de especies tolerantes como intolerantes a la sombra. La menor regeneración observada en el bosque ribereño, también podría estar relacionada a las perturbaciones que tienen lugar durante los periodos de altos flujos de agua. Estas perturbaciones conducen a la fractura de los troncos de los árboles (observación directa), impidiendo el reclutamiento y la regeneración en las zonas ribereñas (Berthelot et al. 2014). Además, la mayor presencia de hojarasca en los bosques de ladera, estaría jugando un rol importante al propiciar una condición adecuada de humedad para la regeneración en este hábitat (Xiong et al. 2003). Por otra parte, una menor luminosidad en el bosque ribereño también explicaría la mayor diversidad de especies trepadoras observada en este hábitat ya que la mayoría de estas especies en los bosques templados de Chile, son sombra tolerantes (Valladares et al. 2011).

Los resultados encontrados son similares a los de otros estudios en los que se comparan la composición, diversidad y estructura entre bosques ribereños y bosques de laderas. Por ejemplo, Suzuki et al. (2002) encontraron mayor riqueza, equitabilidad y diversidad de especies nativas en boques ribereños en comparación con los de las laderas.
Gregory et al. (1991) también concluyeron que la vegetación de las zonas ribereñas exhibe un mayor grado de diversidad estructural y de especies. Estos resultados concuerdan con gradientes de incremento de la diversidad leñosa y de hábitats desde lo más alto de una cuenca hasta la zona ribereña (Corbacho et al. 2003). Sin embargo, contrario a los resultados del presente trabajo, Suzuki et al. (2002) encontraron menor densidad de individuos con dap $>5 \mathrm{~cm}$ en el bosque ribereño, posiblemente debido al mayor grado y frecuencia de perturbaciones naturales que sufren los bosques que evaluaron. Así mismo, contrario a lo esperado, en el bosque ribereño se encontró una menor cobertura y riqueza de especies exóticas asilvestradas en comparación con el bosque de ladera, a pesar de la mayor incidencia de perturbaciones naturales (Hood y Naiman 2000, Sunil et al. 2011). Es posible que la mayor cobertura del estrato 1 -5 $\mathrm{m}$ (sotobosque) encontrada en el bosque ribereño restrinja un mayor ingreso de la radiación solar; y con ello, limita la regeneración de las especies exóticas.

Los resultados de este estudio, hacen evidente la importancia de los bosques ribereños en términos de composición, riqueza, diversidad y estructura de la vegetación. Por ello, diversos autores enfatizan que ellos deben ser prioritarios para la conservación (e.g. Brosofske et al. 1997, Lyon y Gross 2005, Méndez-Toribio et al. 2014). Actualmente, en Chile existen amenazas latentes para los bosques ribereños tales como las plantaciones forestales y las actividades agropecuarias (Altamirano y Lara 2010). La deforestación de esta zona ocasionaría cambios de la temperatura de los cursos de agua, del aire, del suelo y de la humedad (Brosofske et al. 1997), alterando la diversidad o los patrones de regeneración. Además, una reducción de su estratificación o de su cobertura podría incrementar su nivel de invasión (Richardson et al. 2007). En consecuencia, los esfuerzos deben encaminarse a conservar este tipo de vegetación, sobre todo a escala de cuenca, considerando la variabilidad asociada a los flujos de agua (Goebel et al. 2003). Actualmente, la normativa chilena estipula la conservación de una franja de $10 \mathrm{~m}$ a ambos lados de los ríos. Sin embargo, diversos estudios (e.g. Goebel et al. 2003, Langer et al. 2008, Gutierrez y Becerra 2017), sostienen que ello es insuficiente para conservar la diversidad y las funciones ecosistémicas del bosque ribereño. Entonces, solo aumentando al ancho de la franja de protección se podrá asegurar la mantención de su resiliencia a perturbaciones naturales y conservación de su biodiversidad y funciones ecosistémicas.

\section{CONCLUSIONES}

Las hipótesis que se plantean están parcialmente respaldadas por los resultados, puesto que en el bosque ribereño se encuentra diferente composición florística, mayor diversidad de flora vascular (árboles y plantas trepadoras), mayor riqueza de especies nativas, menor nivel de regeneración de árboles y mayor cobertura del estrato arbustivo. 
Sin embargo, contrario a lo esperado, se encuentra mayor cobertura y diversidad de especies exóticas en el bosque de ladera, lo que sugiere una mayor resistencia a la invasión de especies exóticas del bosque ribereño. Así mismo, se observa una mayor cobertura del estrato herbáceo y arbóreo en el bosque de ladera. Estos efectos pueden ser atribuibles a la mayor humedad y perturbaciones naturales en los bosques ribereños en comparación con los bosques de ladera. En general, la mayor diversidad de especies en el bosque ribereño, sugiere que este hábitat contribuye de manera importante a la conservación de la biodiversidad.

Por otra parte, al emplear como réplica a una cuenca, se abarcó una gran área geográfica para incluir diferentes cuencas; pero con ello, se sacrificó el número de parcelas empleadas para caracterizar a cada cuenca. Una alta variabilidad de los datos, potenciada por el bajo número de réplicas (cuencas), puede influir en el bajo poder de la prueba estadística para detectar diferencias de las variables analizadas entre los tipos de bosque. Sin embargo, aun a esta escala de evaluación, los patrones esperados respecto a composición, diversidad o regeneración se mantienen, aunque no para el caso de la cobertura y diversidad de especies exóticas.

\section{AGRADECIMIENTOS}

Este trabajo fue financiado por CONICYT USA, bajo el número de subvención 2012-0011. Ivon Gutierrez fue becaria de maestría de PRONABEC - Perú, durante el tiempo de colección y análisis de datos. Pablo Becerra agradece al proyecto FB 0002-2014.

\section{REFERENCIAS}

Altamirano A, A Lara. 2010. Deforestación en ecosistemas templados de la precordillera andina del centro-sur de Chile. Bosque 31(1): 53-44. DOI: 10.4067/S071792002010000100007.

Berthelot JS, D Saint-Laurent, V Gervais-Beaulac, D Savoie. 2014. Assessing the effects of periodic flooding on the population structure and recruitment rates of riparian tree forests. Water 6: 2614-2633. DOI: 10.3390/w6092614.

Boothroyd IKG, JM Quinn, ER (Lisa) Langer, KJ Costley, G Steward. 2004. Riparian buffers mitigate effects of pine plantation logging on New Zealand streams: 1. Riparian vegetation structure, stream geomorphology and periphyton. Forest Ecology and Management 194(1-3): 199-213. DOI: 10.1016/j.foreco.2004.02.018.

Brosofske KD, J Chen, RJ Naiman, JF Franklin. 1997. Harvesting effects on microclimatic gradients from small streams to uplands in Wastern Washington. Ecological Applications 7(4): 1188-1200. DOI: 10.1890/1051-0761(1997)007[1188:HEOMGF]2.0.CO;2.

Burton ML, LJ Samuelson, S Pan. 2005. Riparian woody diversity and forest structure along an urbal-rural gradient. Urban Ecosystems 8(1): 93-106. DOI: 10.1007/s11252005-1421-6.

Corbacho C, JM Sánchez, E Costillo. 2003. Patterns of structural complexity and human disturbance of riparian vegetation in agricultural landscapes of a Mediterranean area. Agriculture, Ecosystems and Environment 95(2-3): 495-507. DOI: 10.1016/S0167-8809(02)00218-9.

Gajardo R. 1994. La vegetación natural de Chile. Clasificación y distribución geográfica. Santiago, Chile. Editorial Universitaria. $163 \mathrm{p}$.

Goebel C, B Palik, K Pregitzer. 2003. Plant diversity contributions of riparian areas in watersheds of the northern lake states, USA. Ecological Applications 13(6): 1595-1609.

Granados-Sánchez D, MA Hernández-García, GF López-Ríos. 2006. Ecología de las zonas ribereñas. Revista Chapingo. Serie Ciencias Forestales y del Ambiente 12(1): 55-59.

Gregory SV, FJ Swanson, WA McKee, KW Cummins. 1991. An ecosystem perspective of riparian zones. BioScience 41(8): 540-551. DOI: 10.2307/1311607.

Gutierrez IR, PI Becerra. 2017. The effect of native forest replacement by Pinus radiata plantations on riparian plant communities in Chile. Plant Ecology and Diversity 10(1): 1-11. DOI: 10.1080/17550874.2017.1294630.

Harper KA, SE Macdonald. 2001. Structure and composition of riparian boreal forest: New methods for analyzing edge influence. Ecology 82(3): 649-659.

Hood GW, RJ Naiman. 2000. Vulnerability of riparian zones to invasion by exotic vascular plants. Plant Ecology 148: 105114. DOI: 10.1023/A:1009800327334.

Langer ER (Lisa), GA Steward, MO Kimberley. 2008. Vegetation structure, composition and effect of pine plantation harvesting on riparian buffers in New Zealand. Forest Ecology and Management 256(5): 949-957. DOI. 10.1016/j. foreco.2008.05.052.

Lyon J, NM Gross. 2005. Patterns of plant diversity and plantenvironmental relationships across three riparian corridors. Forest Ecology and Management 204(2-3): 267-278. DOI: 10.1016/j.foreco.2004.09.019.

Marticorena C, M Quezada. 1985. Catálogo de la flora vascular de Chile. Gayana (42):1-157.

Méndez-Toribio M, I Zermeño-Hernánez, G Ibarra-Manríquez. 2014. Effect of land use on the structure and diversity of riparian vegetation in the Duero river watershed in Michoacán, Mexico. Plant Ecology 215: 285-296. DOI: 10.1007/ s11258-014-0297-z.

Naiman RJ, H Decamps. 1997. The ecology of interfaces: Riparian zones. Annual Review of Ecology and Systematics 28(102): 621-658. DOI: 10.1146/annurev.ecolsys.28.1.621.

Pauchard A, B Alaback. 2004. Influences of evaluation, land use and landscape context on patterns of alien plat invasions along roadsides in protected areas of South-Central Chile. Conservation Biology 18(1): 238-248.

Ramírez C, F Ferriere, H Figueroa. 1983. Estudio fitosociológico de los bosques pantanosos templados del sur de Chile. Revista Chilena de Historia Natural 56:11-26.

Richardson DM, PM Holmes, KJ Esler, SM Galatowitsch, JC Stromberg, SP Kirkman, P Pysek, RJ Hobbs. 2007. Riparian vegetation: degradation, alien plant invasions, and restoration prospects. Diversity and Distributions 13(1): 126139. DOI: $10.1111 /$ j.1472-4642.2006.00314.x.

Rojas I, P Becerra, N Gálvez, J Laker, C Bonacic, A Hester. 2011. Relationship between fragmentation, degradation and native and exotic species richness in an Andean temperate forest of Chile. Gayana Botanica 68(2): 163-175. 
Sambaré O, F Bognounou, R Witting, A Thiombiano. 2011. Woody species composition, diversity and structure of riparian forests of four watercourses types in Burkina Faso. Journal of Forestry Research 22(2): 145-158. DOI: 10.1007/ s11676-011-0143-2.

Santibáñez F, JM Uribe. 1993. Atlas agroclimático de Chile: regiones sexta, séptima, octava y novena. Santiago, Chile. Ministerio de Agricultura. 99 p.

Sunil C, RK Somashekar, BC Nagaraja. 2011. Impact of anthropogenic disturbances on riparian forest ecology and ecosystem services in Southern India. International Journal of Biodiversity Science, Ecosystem Services and Management 7(4): 273-282. DOI: 10.1080/21513732.2011.631939.

Suzuki W, K Ozumi, T Masaki, K Takahashi, H Daimaru, K Hoshizaki. 2002. Disturbance regimes and community structures of a riparian and an adjacent terrace stand in the Kanumazawa Riparian Research Forest, northern Japan. Forest Ecology and Management 157(1-3): 285-301. DOI: 10.1016/S0378-1127(00)00667-8.

Tropicos (2018). Missouri Botanical Garden (en línea, sitio web). Consultado 25 abr. 2018. Disponible en http://www.tropicos.org.

Valladares F, E Gianoli, A Saldaña. 2011. Climbing plants in a temperate rainforest understorey: Searching for high ligth or coping with deep shade? Annals of Botany 108(2): 231239. DOI: 10.1146/annurev.ecolsys.39.110707.173506.

Xiong S, ME Johansson, FM Hughes, A Hayes, KS Richards, C Nilsson. 2003. Interactive effects of soil moisture, vegetation canopy, plant litter and seed addition on plant diversity in a wetland. Journal of Ecology 91: 976-986. 
Anexo. Especies de plantas registradas en las cuencas de evaluación, tanto en la vegetación del bosque ribereño como en el de ladera, en el centro sur de Chile. *especies exóticas, ** especies con origen desconocido (PIU: Los Piquenes; MIL: El Milico; ARA: Las Arañas; QUE: Los Queñes; MAN: Manqui; SIN: Sin Puerta).

Plant species recorded in the watersheds evaluated, both in the vegetation of the riparian and slope forests, in south-central Chile. *exotic species, **species with unknown origin (PIU: Los Piquenes, MIL: El Milico, ARA: Las Arañas, QUE: Los Queñes, MAN: Manqui, SIN: Sin Puerta).

\begin{tabular}{|c|c|c|c|c|c|c|c|c|c|c|c|c|c|}
\hline \multirow{2}{*}{ Familia } & \multirow{2}{*}{ Nombre científico } & \multicolumn{6}{|c|}{ Vegetación ribereña } & \multicolumn{6}{|c|}{ Vegetación de ladera } \\
\hline & & $\overline{\text { PIU }}$ & MIL & ARA & QUE & MAN & SIN & PIU & MIL & ARA & QUE & MAN & SIN \\
\hline \multicolumn{14}{|l|}{ Árboles } \\
\hline Aextoxicaceae & Aextoxicon punctatum Ruiz et Pav. & 1 & & 1 & 1 & 1 & 1 & 1 & & 1 & 1 & 1 & 1 \\
\hline Myrtaceae & $\begin{array}{l}\text { Amomyrtus luma (Molina) D. Legrand et } \\
\text { Kausel }\end{array}$ & & & 1 & & & & & & & & & \\
\hline Elaeocarpaceae & Aristotelia chilensis (Molina) Stuntz & 1 & 1 & 1 & 1 & 1 & 1 & 1 & 1 & & 1 & & 1 \\
\hline Cupressaceae & $\begin{array}{l}\text { Austrocedrus chilensis (D. Don.) Pic.Serm. et } \\
\text { Bizarri }\end{array}$ & 1 & & & & & & 1 & & & 1 & & \\
\hline Salicaceae & Azara lanceolata Hook. f. & & & 1 & & & & & & 1 & & & \\
\hline Salicaceae & Azara petiolaris (D. Don.) I.M. Johnst. & 1 & 1 & & & & 1 & 1 & 1 & & & & 1 \\
\hline Myrtaceae & $\begin{array}{l}\text { Blepharocalyx cruckshanksii (Hook. et Arn.) } \\
\text { Nied. }\end{array}$ & & & 1 & 1 & 1 & & & & & & & \\
\hline Cunoniaceae & Caldcluvia paniculata (Cav.) D. Don & & & 1 & & 1 & & & & 1 & & & \\
\hline Cardiopteridaceae & Citronella mucronata (Ruiz et Pav.) D. Don & 1 & & 1 & 1 & 1 & & & & & 1 & 1 & \\
\hline Lauraceae & Cryptocarya alba (Molina) Looser & 1 & & & 1 & 1 & 1 & 1 & & & 1 & 1 & 1 \\
\hline Winteraceae & Drimys winteri J.R. Forst. et G. Forst. & 1 & & 1 & 1 & & 1 & & & 1 & & & \\
\hline Cunoniaceae & Eucryphia cordifolia Cav. & & & & & & & & & 1 & & & \\
\hline Oleaceae & *Fraxinus excelsior $\mathrm{L}$. & & & & & 1 & & & & & & 1 & \\
\hline Proteaceae & Gevuina avellana Molina & & & 1 & 1 & 1 & 1 & & & 1 & 1 & 1 & 1 \\
\hline Rosaceae & Kageneckia oblonga Ruiz et Pav. & 1 & & & & & & & & & & & \\
\hline Monimiaceae & Laurelia sempervirens (Ruiz et Pav.) Tul. & 1 & & 1 & & 1 & 1 & 1 & & 1 & 1 & 1 & \\
\hline Myrtaceae & Legrandia concinna (Phil.) Kausel & & & & 1 & & & & & & & & \\
\hline Anacardiaceae & Lithraea caustica (Molina) Hook. et Arn. & 1 & & & & & & 1 & & & & 1 & 1 \\
\hline Proteaceae & Lomatia dentata (Ruiz et Pav.) R.Br. & 1 & & 1 & 1 & 1 & 1 & 1 & & 1 & 1 & 1 & 1 \\
\hline Proteaceae & Lomatia ferruginea (Cav.) R.Br. & & & 1 & & & & & & 1 & & & \\
\hline Proteaceae & Lomatia hirsuta (Lam.) Diels. & 1 & & & 1 & & & 1 & & & 1 & & \\
\hline Myrtaceae & Luma apiculata (DC.) Burret & 1 & & & 1 & 1 & 1 & 1 & & 1 & 1 & 1 & 1 \\
\hline Myrtaceae & Luma chequen (Molina) A. Gray & & & & & & 1 & & & & & & \\
\hline Celastraceae & Maytenus boaria Molina & 1 & 1 & & 1 & & & 1 & & & 1 & & 1 \\
\hline Myrtaceae & Myrceugenia obtusa (DC.) O. Berg. & & & & & 1 & & & & & & & \\
\hline Myrtaceae & $\begin{array}{l}\text { Myrceugenia planipes (Hook. et Arn.) O. } \\
\text { Berg. }\end{array}$ & & & 1 & & & & & & & & & \\
\hline Nothofagaceae & Nothofagus alpina (Poepp. et Endl.) Oerst. & & & & & & & & & 1 & 1 & & \\
\hline Nothofagaceae & Nothofagus dombeyi (Mirb.) Oerst. & 1 & 1 & & 1 & & & & 1 & 1 & & & \\
\hline Nothofagaceae & Nothofagus glauca (Phil.) Krasser & 1 & & & & & & 1 & & & & & 1 \\
\hline Nothofagaceae & Nothofagus obliqua (Mirb.) Oerst. & 1 & 1 & & 1 & & 1 & & 1 & & 1 & 1 & 1 \\
\hline Nothofagaceae & Nothofagus pumilio (Poepp. et Endl.) Krasser & & & & & & & & 1 & & & & \\
\hline Fabaceae & Otholobium glandulosum (L.) J.W. Grimes & & & & & & 1 & & & & & & 1 \\
\hline Lauraceae & Persea lingue (Ruiz et Pav.) Nees & & & 1 & 1 & 1 & 1 & & & 1 & 1 & 1 & 1 \\
\hline
\end{tabular}




\begin{tabular}{|c|c|c|c|c|c|c|c|c|c|c|c|c|c|}
\hline Monimiaceae & Peumus boldus Molina & 1 & & & & & 1 & 1 & & & & & 1 \\
\hline Podocarpaceae & Podocarpus salignus D. Don & & & & 1 & & & & & & 1 & & \\
\hline Quillajaceae & Quillaja saponaria Molina & 1 & & & & & 1 & & & & 1 & & \\
\hline Araliaceae & Raukaua laetevirens (Gay) Frodin. & & & 1 & & & & & & 1 & & & \\
\hline Cunoniaceae & Weinmannia trichosperma Cav. & & & 1 & & & & & & 1 & & & \\
\hline \multicolumn{14}{|l|}{ Arbustos } \\
\hline Asteraceae & $\begin{array}{l}\text { Acrisione denticulata (Hook. et Arn.) B. } \\
\text { Nord. }\end{array}$ & 1 & 1 & & & & & 1 & & & & 1 & \\
\hline Fabaceae & Adesmia elegans Clos & & & & & & & 1 & & & & & \\
\hline Phytolaccaceae & Anisomeria sp. & & 1 & & & & & & 1 & & & & \\
\hline Asteraceae & $\begin{array}{l}\text { Ageratina glechonophylla (Less.) R.M.King } \\
\text { et H.Rob. }\end{array}$ & 1 & & & & & & 1 & & & & & \\
\hline Salicaceae & Azara serrata Ruiz et Pav. & 1 & 1 & & & & & & 1 & & 1 & & 1 \\
\hline Asteraceae & Baccharis sp. & & 1 & & & & 1 & & 1 & & & & 1 \\
\hline Asteraceae & Baccharis neaei DC. & & 1 & & & & & & 1 & & & & \\
\hline Asteraceae & Baccharis obovata Hook. et Arn. & 1 & & & & & & 1 & & & & & \\
\hline Asteraceae & Baccharis racemosa (Ruiz et Pav.) DC. & & & & & & & 1 & & & & & \\
\hline Asteraceae & Baccharis sagittalis (Less.) DC. & & 1 & & & 1 & & & & & & 1 & \\
\hline Asteraceae & Baccharis salicifolia (Ruiz et Pav.) Pers. & 1 & & & & & & & & & & & 1 \\
\hline Berberidaceae & Berberis sp. & & & & & & & & & 1 & & & \\
\hline Berberidaceae & Berberis rotundifolia Poepp. et Endl. & & 1 & & 1 & & 1 & & & & 1 & & 1 \\
\hline Buddlejaceae & Buddleja globosa Hope & & 1 & & & & & & & & & & \\
\hline Calceolariaceae & Calceolaria andina Benth. & & 1 & & & & & & 1 & & & & \\
\hline Calceolariaceae & Calceolaria Cf. talcana Grau et Ehr. Bayer & 1 & & & & & & & & & & & \\
\hline Poaceae & Chusquea culeou E. Desv. & 1 & & & & & & 1 & & & & & \\
\hline Poaceae & Chusquea quila Kunth & & 1 & 1 & 1 & 1 & 1 & & 1 & 1 & 1 & 1 & 1 \\
\hline Rhamnaceae & Colletia hystrix Clos & 1 & 1 & & 1 & & & 1 & 1 & & & & \\
\hline Euphorbiaceae & Colliguaja dombeyana A. Juss. & 1 & & & & & 1 & 1 & & & & & \\
\hline Euphorbiaceae & Colliguaja integerrima Gillies et Hook. & & & & & & 1 & & & & & & 1 \\
\hline Euphorbiaceae & Colliguaja odorifera Molina & 1 & & & & & 1 & & & & & & \\
\hline Coriariaceae & Coriaria ruscifolia $\mathrm{L}$. & 1 & & & & & & & & & & & \\
\hline Escalloniaceae & Escallonia cfr. revoluta (Ruiz et Pav.) Pers. & & & & 1 & & 1 & & & & & & 1 \\
\hline Escalloniaceae & Escallonia rubra (Ruiz et Pav.) Pers. & 1 & & 1 & & & & 1 & & & & & \\
\hline Onagraceae & Fuchsia magellanica Lam. & & & 1 & & & & & & & & & \\
\hline Ericaceae & Gaultheria mucronata (L. f.) Hook. et Arn. & & 1 & 1 & 1 & & & 1 & 1 & 1 & & & \\
\hline Ericaceae & Gaultheria pumila (L. f.) D. J. Middleton & & 1 & & & & & & & & & & \\
\hline Asteraceae & Haplopappus sp. & 1 & & & & & & 1 & & & & & \\
\hline Scrophulariaceae & Jovellana violacea (Cav.) G. Don. & & & & & 1 & & & & & & & \\
\hline Scrophulariaceae & Jovellana punctata Ruiz et Pav. & & & 1 & & & & & & & & & \\
\hline Celastraceae & Maytenus disticha (Hook. f.) Urb. & & 1 & & & 1 & & & 1 & & & 1 & \\
\hline Celastraceae & Maytenus magellanica (Lam.) Hook. f. & & 1 & & & & & & 1 & & & & \\
\hline Misodendraceae & Misodendrum punctulatum Banks ex DC. & & & & & & & & 1 & & & & \\
\hline Asteraceae & Podanthus mitiqui Lindl. & 1 & & & & & & 1 & & & & & \\
\hline
\end{tabular}




\begin{tabular}{|c|c|c|c|c|c|c|c|c|c|c|c|c|c|}
\hline Grossulariaceae & Ribes punctatum Ruiz et Pav. & 1 & 1 & & & & & 1 & 1 & & & & 1 \\
\hline Grossulariaceae & Ribes magellanicum Poir. & & 1 & & & & & & & & & & \\
\hline Grossulariaceae & Ribes valdivianum Phil. & & & & & & & & & & 1 & & \\
\hline Verbenaceae & Rhaphithamnus spinosus (Juss.) Moldenke & & & 1 & 1 & 1 & 1 & & & 1 & 1 & & \\
\hline Rosaceae & *Rosa rubiginosa L. & & & & 1 & & & & & & 1 & & 1 \\
\hline Rosaceae & *Rubus ulmifolius Schott & 1 & & & 1 & 1 & 1 & & & & 1 & 1 & 1 \\
\hline Anacardiaceae & $\begin{array}{l}\text { Schinus patagonicus (Phil.) I.M. Johnst. ex } \\
\text { Cabrera }\end{array}$ & & 1 & & & & & & 1 & & & & \\
\hline Boraginaceae & $\begin{array}{l}\text { Selkirkia pauciflora (Ruiz et Pav.) Holstein et } \\
\text { Weigend }\end{array}$ & & & & & 1 & & & & & & & \\
\hline Solanaceae & Solanum crispum Ruiz et Pav. & & & 1 & & 1 & & & & & & & \\
\hline Fabaceae & Sophora macrocarpa $\mathrm{Sm}$. & 1 & & & 1 & & 1 & 1 & & & 1 & & 1 \\
\hline Fabaceae & Sophora microphylla Aiton & & & & 1 & & & & & & & & \\
\hline Lamiaceae & Teucrium bicolor $\mathrm{Sm}$. & & 1 & & & & & & & & & & \\
\hline Myrtaceae & Ugni molinae Turcz. & & & 1 & & & & & & 1 & & & 1 \\
\hline Violaceae & Viola portalesia Gay & 1 & & 1 & 1 & & 1 & 1 & & & 1 & & 1 \\
\hline Vivianiaceae & Viviania Cf. crenata (Hook.) G.Don & 1 & & & & & & & & & & & \\
\hline Vivianiaceae & Wendtia gracilis Meyen & 1 & & & & & & & & & & & \\
\hline \multicolumn{14}{|c|}{ Hierbas anuales o perennes } \\
\hline $\mathrm{NN}$ & $* * N N \_1$ & & & & & & & & & & 1 & & \\
\hline Rosaceae & Acaena argentea Ruiz et Pav. & & 1 & & & & & & & & 1 & & \\
\hline Pteridaceae & Adiantum chilense Kaulf. & 1 & & 1 & 1 & 1 & 1 & 1 & & & & 1 & 1 \\
\hline Pteridaceae & Adiantum sulphureum Kaulf. & & 1 & & & & & & & & & & \\
\hline Poaceae & *Cf. Agrostis & & & & & & & 1 & & & & & \\
\hline Alstroemeriaceae & Alstroemeria sp1. & 1 & & & & & & & & & & & \\
\hline Alstroemeriaceae & Alstroemeria sp2. & & 1 & & & & & & 1 & & & & \\
\hline Ranunculaceae & Anemone sp. & 1 & & & & & & & & & & & \\
\hline Apiaceae & Azorella spinosa (Ruiz et Pav.) Pers. & & & & & & & 1 & 1 & & & & \\
\hline Blechnaceae & Blechnum chilense (Kaulf.) Mett. & 1 & 1 & & & 1 & & 1 & & & & & \\
\hline Blechnaceae & Blechnum hastatum Kaulf. & 1 & & 1 & 1 & 1 & 1 & & & 1 & 1 & 1 & 1 \\
\hline Blechnaceae & Blechnum mochaenum G. Kunkel & & & & & 1 & & & & & & 1 & \\
\hline Cyperaceae & Carex sp. & & & & & & 1 & & & & & & 1 \\
\hline Cyperaceae & Carex Cf. excelsa Peopp. ex Kunth & & & & 1 & & & & & & 1 & & \\
\hline Cyperaceae & Carex Cf. setifolia Kunze ex Kunth & 1 & & & & & & 1 & & & & & \\
\hline Pteridaceae & Cheilanthes hypoleuca (Kunze) Mett. & 1 & & & & & & & & & & & \\
\hline Euphorbiaceae & Chiropetalum tricuspidatum (Lam.) A. Juss. & & & & & 1 & 1 & & & & & & \\
\hline Asteraceae & *Cirsium vulgare (Savi) Ten. & & & & & & & & & & 1 & & \\
\hline Asteraceae & Coniza sp. & & & & & & & & & & 1 & & \\
\hline Boraginaceae & ${ }^{*}$ Echium vulgare $\mathrm{L}$. & & & & & & & & & & 1 & & \\
\hline Apiaceae & $\begin{array}{l}\text { Eryngium paniculatum Cav. et Dombey ex F. } \\
\text { Delaroche }\end{array}$ & 1 & & 1 & & & & 1 & 1 & 1 & & & 1 \\
\hline Equisetaceae & Equisetum bogotense Kunth & 1 & & & 1 & 1 & 1 & 1 & & & & & \\
\hline Bromeliaceae & Fascicularia bicolor (Ruiz et Pav.) Mez & & & 1 & & & & & & & & & \\
\hline
\end{tabular}




\begin{tabular}{|c|c|c|c|c|c|c|c|c|c|c|c|c|}
\hline Francoaceae & Francoa appendiculata Cav. & 1 & & & & 1 & & & & & & \\
\hline Rubiaceae & Galium hypocarpium (L.) Endl. ex Griseb. & 1 & & & 1 & & 1 & 1 & & 1 & & 1 \\
\hline Asteraceae & Gamochaeta sp. & & 1 & & & & & & & & & \\
\hline Asteraceae & Gamochaeta americana (Mill.) Wedd. & & & 1 & & & & & & & & \\
\hline Asteraceae & Gamochaeta $\mathrm{cfr}$ chamissonis (DC.) Cabrera & & & & & & & 1 & & & & \\
\hline Asteraceae & Gamochaeta chamissonis (DC.) Cabrera & 1 & & & & & & 1 & & & & \\
\hline Geraniaceae & Geranium core-core Steud. & & & & & & & & & 1 & & \\
\hline Geraniaceae & Geranium robertianum L. & 1 & & & & & & & & & & \\
\hline Geraniaceae & Geranium sessiliflorum Cav. & & 1 & & & & & & & & & \\
\hline Asteraceae & Gnaphalium sp. & & & 1 & & & & & & & & \\
\hline Bromeliaceae & Greigia sphacelata (Ruiz et Pav.) Regel & & & & & 1 & & & & & 1 & \\
\hline Gunneraceae & Gunnera tinctoria (Molina) Mirb. & & & 1 & 1 & & & & & & & \\
\hline Asteraceae & *Hypochaeris radicata $\mathrm{L}$. & 1 & & 1 & & & 1 & & & & & \\
\hline Hypericaceae & Hypericum caespitosum Cham. et Schltdl. & & & & & & & & & 1 & & \\
\hline Hypericaceae & *Hypericum perforatum $\mathrm{L}$. & 1 & & & & & & & & & & \\
\hline Asteraceae & *Lactuca virosa $\mathrm{L}$. & 1 & & & & & & 1 & & & & \\
\hline Iridaceae & Libertia sessiliflora (Poepp.) Skottsb. & 1 & & & & 1 & & 1 & & & 1 & \\
\hline Iridaceae & Libertia tricocca Phil. & & & 1 & & & & & & & & \\
\hline Loasaceae & Loasa tricolor Ker Gawl & & & & & 1 & & & & & & \\
\hline Poaceae & *Cf. Lolium multiflorum Lam. & & & & & & & & & 1 & & \\
\hline Dicksoniaceae & $\begin{array}{l}\text { Lophosoria quadripinnata (J.F. Gmel.) C. } \\
\text { Chr. }\end{array}$ & & & 1 & & 1 & & & 1 & & & \\
\hline Fabaceae & *Medicago orbicularis (L.) Bartal. & & & & & & & & & 1 & & \\
\hline Dryopteridaceae & $\begin{array}{l}\text { Megalastrum spectabile (Kaulf.) A.R. Sm. et } \\
\text { R.C. Moran }\end{array}$ & & & 1 & & 1 & & & & & & \\
\hline Apiaceae & Osmorhiza chilensis Hook. et Arn. & & & & 1 & & & & & 1 & & \\
\hline Oxalidaceae & $* *$ Oxalis sp. & & 1 & & & & & & & & & \\
\hline Oxalidaceae & Oxalis Cf. dumetorum Barnéoud & 1 & & & 1 & & 1 & 1 & & 1 & & \\
\hline Hydrophyllaceae & Phacelia secunda J. F. Gmel. & 1 & & & & & & & & & & \\
\hline Aspleniaceae & Pleurosorus papaverifolius (Kunze) Fée & 1 & & & & & & & & & & \\
\hline Poaceae & *Poa sp. & & 1 & & & & & & 1 & & & \\
\hline Polypodiaceae & Polypodium feuillei Bertero & 1 & & & & & & & & & & \\
\hline Dryopteridaceae & Polystichum chilense (H. Christ) Diels & 1 & & & & & 1 & 1 & & & & \\
\hline Bromeliaceae & $\begin{array}{l}\text { Puya coerulea var. violacea (Brongn.) L.B. } \\
\text { Sm. et Looser }\end{array}$ & 1 & & & & & & 1 & & & & \\
\hline Santalaceae & Quinchamalium chilense Molina & & & & & & & & 1 & & & \\
\hline Rosaceae & Rubus geoides Sm. & & & & & & & & & 1 & & \\
\hline Polygonaceae & *Rumex acetosella L. & 1 & & & & & & & & 1 & & \\
\hline Rosaceae & *Sanguisorba minor Scop. & & & & & & & & & 1 & & \\
\hline Asteraceae & *Senecio sp. & & & & & & & 1 & & & & \\
\hline Iridaceae & Sisyrinchium sp. & & 1 & & & & & & 1 & & & \\
\hline Amaryllidaceae & Solaria miersioides Phil. & & & & & 1 & & & & & & \\
\hline Fabaceae & *Trifolium repens L. & & & & & & & & & 1 & & \\
\hline
\end{tabular}




\begin{tabular}{|c|c|c|c|c|c|c|c|c|c|c|c|c|c|}
\hline Cyperaceae & Uncinia sp. & & & 1 & & & & & & & & & \\
\hline Cyperaceae & Uncinia phleoides (Cav.) Pers. & & & 1 & & & & & & & & & \\
\hline Caprifoliaceae & Valeriana sp1. & 1 & & & & & & & & & & & \\
\hline Caprifoliaceae & Valeriana sp2. & & & & & & & & & & 1 & & \\
\hline Caprifoliaceae & Valeriana Cf. laxiflora DC. & & & & & & & 1 & & & & & \\
\hline Fabaceae & Vicia sp. & 1 & & & & & & & & & & & \\
\hline Violaceae & Viola sp. & & & 1 & & & & & & 1 & & & \\
\hline Asteraceae & cfr. Xanthium spinosum L. & & & & & & & 1 & & & & & \\
\hline $\mathrm{NN}$ & $* * \mathrm{NN} \_2$ & 1 & & & & & & & & & & & \\
\hline $\mathrm{NN}$ & $* * \mathrm{NN} \_3$ & & & & 1 & & & & & & & & \\
\hline $\mathrm{NN}$ & **NN_4 & & & & & 1 & & & & & & & \\
\hline $\mathrm{NN}$ & $* * \mathrm{NN} \_5$ & & & & & & 1 & & & & & & 1 \\
\hline Orchidaceae & Orquídea & 1 & & & 1 & & & & & & & & \\
\hline \multicolumn{14}{|l|}{ Plantas trepadoras } \\
\hline Gesneriaceae & Asteranthera ovata (Cav.) Hanst. & & & 1 & & & & & & & & & \\
\hline Berberidopsidaceae & Berberidopsis corallina Hook.f. & & & 1 & & & & & & 1 & & & \\
\hline Alstroemeriaceae & Bomarea salsilla (L.) Herb. & 1 & & 1 & & 1 & 1 & & & & & & 1 \\
\hline Lardizabalaceae & Boquila trifoliolata (DC.) Decne. & & & 1 & 1 & 1 & 1 & & & 1 & 1 & & 1 \\
\hline Bignoniaceae & Campsidium valdivianum (Phil.) Skottsb. & & & 1 & & & & & & & & & \\
\hline Vitaceae & Cissus striata Ruiz et Pav. & 1 & & & 1 & 1 & 1 & 1 & & & 1 & 1 & 1 \\
\hline Apocynaceae & $\begin{array}{l}\text { Cynanchum lancifolium Hook. et Arn., hom. } \\
\text { Illeg. }\end{array}$ & & & 1 & & & & & & 1 & & & \\
\hline Dioscoreaceae & Dioscorea sp. & & & 1 & & & & & & & & & \\
\hline Dioscoreaceae & Dioscorea auriculata Poepp. & & & & & & 1 & & & & & & 1 \\
\hline Dioscoreaceae & Dioscorea humifusa Poepp. & & & & & & & & & & & 1 & \\
\hline Dioscoreaceae & Dioscorea humilis Bertero ex Colla & 1 & & & & & & & & & & & \\
\hline Herreriacae & Herreria stellata Ruiz et Pav. & & & & & 1 & & & & & & & \\
\hline Hydrangeaceae & $\begin{array}{l}\text { Hydrangea serratifolia (Hook. et Arn.) F. } \\
\text { Phil. }\end{array}$ & 1 & 1 & 1 & 1 & 1 & 1 & & & & 1 & 1 & 1 \\
\hline Philesiaceae & Lapageria rosea Ruiz et Pav. & & & 1 & 1 & 1 & 1 & & & 1 & 1 & 1 & \\
\hline Lardizabalaceae & Lardizabala biternata Ruiz et Pav. & 1 & & & 1 & 1 & 1 & 1 & & 1 & & 1 & 1 \\
\hline Eremolepidaceae & Lepidoceras chilense (Molina) Kuijt & & & & 1 & & & & & & & & \\
\hline Luzuriagaceae & Luzuriaga radicans Ruiz et Pav. & & & 1 & 1 & 1 & 1 & & & & & 1 & \\
\hline Gesneriaceae & Mitraria coccinea Cav. & & & 1 & & 1 & & & & & & 1 & \\
\hline Polygonaceae & Muehlenbeckia hastulata (Sm.) I.M. Johnst. & 1 & & & & 1 & & & & & & & 1 \\
\hline Asteraceae & Mutisia sp. & & & 1 & & & & & & & & & \\
\hline Asteraceae & Mutisia brachyantha Phil. & & & & & & & 1 & & & & & \\
\hline Asteraceae & Mutisia decurrens Cav. & & & & & & & & 1 & & & & \\
\hline Asteraceae & Proustia pyrifolia DC. & 1 & & & & 1 & & 1 & & & & 1 & 1 \\
\hline Gesneriaceae & $\begin{array}{l}\text { Sarmienta scandens (J. D. Brandis ex Molina) } \\
\text { Pers. }\end{array}$ & & & 1 & & 1 & & & & & & 1 & \\
\hline Loranthaceae & Tristerix corymbosus (L.) Kuijt & & & & & & & 1 & & & 1 & & \\
\hline Tropaeolaceae & Tropaeolum tricolor Sweet & & & & & 1 & & & & & & 1 & \\
\hline Total & & 74 & 34 & 52 & 44 & 48 & 44 & 51 & 25 & 29 & 49 & 31 & 41 \\
\hline
\end{tabular}


